Journal of Nepal Mathematical Society (JNMS), Vol. 1, Issue 2 (2018); Attaullah

\title{
Solving Some Linear and Nonlinear PDEs Using Laplace Adomian Decomposition Method
}

\author{
Attaullah \\ Department of Mathematics, University of Malakand, Chakdara Dir(L), KPK, Pakistan \\ Correspondence to: Attaullah, Email: Uatta517@gmail.com
}

\begin{abstract}
In this paper, Laplace Adomian decomposition method (LADM) is applied to solve linear and nonlinear partial differential equations (PDEs). With the help of proposed method, we handle the approximated analytical solutions to some interesting classes of PDEs including nonlinear evolution equations, Cauchy reaction-diffusion equations and the Klien-Gordon equations.
\end{abstract}

Keywords: Laplace Adomian decomposition method, Nonlinear Fisher's equation, Evolution equations, Cauchy reaction-diffusion equations, Klien-Gordon equations.

\section{Introduction}

Nonlinear differential equations play very important role to model real world problems in various fields of physics, engineering, chemistry, dynamics, fluid mechanics, etc, see for detail [1, 4]. Most of the nonlinear phenomena of our real-life problems can be modelled by using partial differential equations. To find the exact solutions to the nonlinear problems is not an easy task and in some situations it is quiet difficult. In this regard, researchers have developed various iterative techniques to find numerical and analytical solutions of nonlinear and linear partial differential equations. Thanks to these tools, we find the approximated solutions and obtain useful information about the solutions of the problems. A variety of powerful methods to find numerical solutions of nonlinear PDEs has been presented, such as the homotopy analysis method (HAM) 7, 14, Adomain decomposition method (ADM) 12, homotopy perturbation method (HPM), Fourier transform method(FTM) 21, Laplace and natural transform methods [17, 18, variational iteration method (VIM) [14, etc. In last few decades, it has been proved that Laplace transform coupled with Adomian decomposition and perturbation method provides excellent tool to find numerical solutions of large number of PDEs and ODEs. To find the numerically approximated solutions of nonlinear PDEs, we use Laplace Adomian decomposition techniques in this paper. The Laplace Adomian decomposition method (LADM) is modified form of Adomian decomposition method which is used for solving linear and nonlinear PDEs, see 13, 19, 5, 11. The adapted numerical procedure basically illustrates how the Laplace transform may be implemented to approximate the solutions of the nonlinear partial differential equations by manipulating the decomposition method. The proposed techniques provide a useful way to establish an analytic treatment for linear and nonlinear PDEs. The proposed method is a semi-analytical method to obtain the approximated solutions of linear and nonlinear types of differential equations. Here, we notice that the proposed method provides an excellent approximation solutions for a large numbers of algebraic, integral, functional, and partial differential equations, see [2, 3, 4, 19. The Laplace Adomian decomposition method (LADM) has significant applications to handle the analytical solutions of real world problems of physics, biology, chemistry, and engineering, fluid mechanics, see [4], etc.

\section{Preliminaries}

In this section, we consider basic definition of the Laplace transform needed throughout this paper. For the function $\Phi(x, t)$ defined for $x \in[a, b]$ and $t>0$, the Laplace transform of $\Phi(x, t)$ is defined as

$$
\mathcal{L}[\Phi(x, t)]=\int_{0}^{\infty} \exp (-s t) \Phi(x, t) d t, s>0 .
$$


Solving Some Linear and Nonlinear PDEs Using Laplace Adomian Decomposition Method

\section{Procedure of Laplace Adomian Decomposition Method}

To explain the Laplace Adomian decomposition method, we consider a general partial differential equation of the type

$$
\begin{aligned}
& \mathbf{L} \phi(x, t)+\mathbf{R} \phi(x, t)+\mathbf{N} \phi(x, t)=\gamma(x, t), \\
& \text { with initial conditions } \\
& \phi(x, 0)=\alpha(x), \phi_{t}(x, 0)=\beta(x) .
\end{aligned}
$$

Where $\mathbf{L}$ is considered here as the second order partial differential operator in $x, \mathbf{R}$ is the remaining linear operator, $\mathbf{N}$ represent a general nonlinear differential operator, and $\gamma(x, t)$ is the source term.

By applying Laplace transform to both side of equation (1), and using given initial conditions, we obtain

$$
\mathcal{L}[\phi(x, t)]=\frac{1}{s} \alpha(x)+\frac{1}{s^{2}} \beta(x)-\frac{1}{s^{2}}[\mathcal{L}(\mathbf{R} \phi(x, t))-\mathcal{L}(\mathbf{N} \phi(x, t))+\mathcal{L}(\gamma(x, t))] .
$$

Applying inverse Laplace transform to (2), it gives

$$
\phi(x, t)=\alpha(x)+t \beta(x)-\mathcal{L}^{-1}\left[\frac{1}{s^{2}} \mathcal{L}(\mathbf{R} \phi(x, t))\right]-\mathcal{L}^{-1}\left[\frac{1}{s^{2}} \mathcal{L}(\mathbf{N} \phi(x, t))\right]+\mathcal{L}^{-1}\left[\frac{1}{s^{2}} \mathcal{L}(\gamma(x, t))\right] .
$$

The Laplace Adomian decomposition method decomposes the unknown functions $\phi(x, t)$ by an infinite series of components as

$$
\phi(x, t)=\sum_{n=0}^{\infty} \phi_{n}(x, t)
$$

and the nonlinear term by Adomian polynomial as

$$
\mathbf{N} \phi(x, t)=\sum_{n=0}^{\infty} P_{n}(x, t)
$$

where

$$
P_{n}(x, t)=\left.\frac{1}{n !}\left[\frac{d^{n}}{d \lambda^{n}}\left(\mathbf{N} \sum_{i=0}^{\infty} \lambda^{i} \phi_{i}\right)\right]\right|_{\lambda=0} .
$$

Putting equation (4) and equation (5) in equation (3), it gives

$$
\begin{gathered}
\sum_{n=0}^{\infty} \phi_{n}(x, t)=\alpha(x)+t \beta(x)-\mathcal{L}^{-1}\left[\frac{1}{s^{2}} \mathcal{L}\left(\mathbf{R} \sum_{n=0}^{\infty} \phi_{n}(x, t)\right)\right] \\
-\mathcal{L}^{-1}\left[\mathcal{L}\left(\frac{1}{s^{2}} \sum_{n=0}^{\infty} P_{n}(x, t)\right)\right]+\mathcal{L}^{-1}\left[\frac{1}{s^{2}} \mathcal{L}(\gamma(x, t))\right] .
\end{gathered}
$$

Putting values for $n=0,1,2, \ldots$, then comparing both sides, we have from (7)

$$
\begin{aligned}
& \phi_{0}(x, t)=\alpha(x)+t \beta(x)+\mathcal{L}^{-1}\left[\frac{1}{s^{2}} \mathcal{L}[\gamma(x, t)]\right] \\
& \phi_{1}(x, t)=-\mathcal{L}^{-1}\left[\frac{1}{s^{2}} \mathcal{L}\left[\mathbf{R} \phi_{0}(x, t)\right]\right]-\mathcal{L}^{-1}\left[\frac{1}{s^{2}} \mathcal{L}\left[P_{0}(x, t)\right]\right], \\
& \phi_{2}(x, t)=-\mathcal{L}^{-1}\left[\frac{1}{s^{2}} \mathcal{L}\left[\mathbf{R} \phi_{1}(x, t)\right]\right]-\mathcal{L}^{-1}\left[\frac{1}{s^{2}} \mathcal{L}\left[P_{1}(x, t)\right]\right], \\
& \phi_{3}(x, t)=-\mathcal{L}^{-1}\left[\frac{1}{s^{2}} \mathcal{L}\left[\mathbf{R} \phi_{2}(x, t)\right]\right]-\mathcal{L}^{-1}\left[\frac{1}{s^{2}} \mathcal{L}\left[P_{2}(x, t)\right]\right]
\end{aligned}
$$

and so on. Generalizing this result

$$
\sum_{n=0}^{\infty} \phi_{n+1}(x, t)=-\mathcal{L}^{-1}\left[\frac{1}{s^{2}} \mathcal{L}\left[\mathbf{R} \sum_{n=0}^{\infty} \phi_{n}(x, t)\right]\right]-\mathcal{L}^{-1}\left[\frac{1}{s^{2}} \mathcal{L}\left[\sum_{n=0}^{\infty} P_{n}(x, t)\right]\right], \text { for } n \geq 0 .
$$




\section{Applications of Laplace Adomian Decomposition Method to Different Types PDEs}

Example 4.1. Consider the nonlinear PDE [20]

$$
\begin{aligned}
& \qquad \phi_{t t}+\phi_{x}^{2}-\phi^{2}+\phi=t \exp (-x), \\
& \text { with initial conditions } \\
& \qquad(x, 0)=0, \phi_{t}(x, 0)=\exp (-x) .
\end{aligned}
$$

Taking Laplace transform on both sides of equation (10) and using initial conditions (10), it gives

$$
\mathcal{L}[\phi(x, t)]=\frac{1}{s^{2}} \exp (-x)-\frac{1}{s^{2}} \mathcal{L}\left[\phi_{x}^{2}\right]+\frac{1}{s^{2}} \mathcal{L}\left[\phi^{2}\right]-\frac{1}{s^{2}} \mathcal{L}[\phi]+\frac{1}{s^{2}} \mathcal{L}[t \exp (-x)] .
$$

Applying inverse Laplace transform to 11, we get

$$
\phi(x, t)=t \exp (-x)-\mathcal{L}^{-1}\left[\frac{1}{s^{2}} \mathcal{L}\left[\phi_{x}^{2}\right]\right]+\mathcal{L}^{-1}\left[\frac{1}{s^{2}} \mathcal{L}\left[\phi^{2}\right]\right]-\mathcal{L}^{-1}\left[\frac{1}{s^{2}} \mathcal{L}[\phi]\right]+\mathcal{L}^{-1}\left[\frac{1}{s^{2}} \mathcal{L}[t \exp (-x)]\right] .
$$

We write $\phi(x, t)$ in the form of an infinite series as

$$
\phi(x, t)=\sum_{n=0}^{\infty} \phi_{n}(x, t)
$$

and the nonlinear terms $\phi^{2}$ and $\phi_{x}^{2}$ be defined by Adomian polynomials as

$$
\begin{aligned}
& \phi^{2}=\sum_{n=0}^{\infty} A_{n}(x, t), \\
& \phi_{x}^{2}=\sum_{n=0}^{\infty} B_{n}(x, t),
\end{aligned}
$$

where

$$
\begin{aligned}
& A_{n}(x, t)=\left.\frac{1}{n !} \frac{d^{n}}{d \lambda^{n}}\left[\sum_{i=0}^{\infty}\left(\lambda^{i} \phi_{i}\right)^{2}\right]\right|_{\lambda=0} . \\
& B_{n}(x, t)=\left.\frac{1}{n !} \frac{d^{n}}{d \lambda^{n}}\left[\sum_{i=0}^{\infty}\left(\lambda^{i} \phi_{i, x}\right)^{2}\right]\right|_{\lambda=0} .
\end{aligned}
$$

Putting equations (13), 14) and 15) in 12, we get

$$
\begin{aligned}
& \sum_{n=0}^{\infty} \phi_{n}(x, t)=t \exp (-x)-\mathcal{L}^{-1}\left[\frac{1}{s^{2}} \mathcal{L}\left[\sum_{n=0}^{\infty} B_{n}(x, t)\right]\right]+\mathcal{L}^{-1}\left[\frac{1}{s^{2}} \mathcal{L}\left[\sum_{n=0}^{\infty} A_{n}(x, t)\right]\right] \\
&-\mathcal{L}^{-1}\left[\frac{1}{s^{2}} \mathcal{L}\left[\sum_{n=0}^{\infty} \phi_{n}(x, t)\right]\right]+\mathcal{L}^{-1}\left[\frac{1}{s^{2}} \mathcal{L}[t \exp (-x)]\right] .
\end{aligned}
$$

Inserting values for $n=0,1,2, \ldots$, we obtain

$$
\begin{aligned}
& \phi_{0}(x, t)=t \exp (-x) \\
& \phi_{1}(x, t)=0
\end{aligned}
$$

All the other terms are vanishing for $n \geq 2$. 


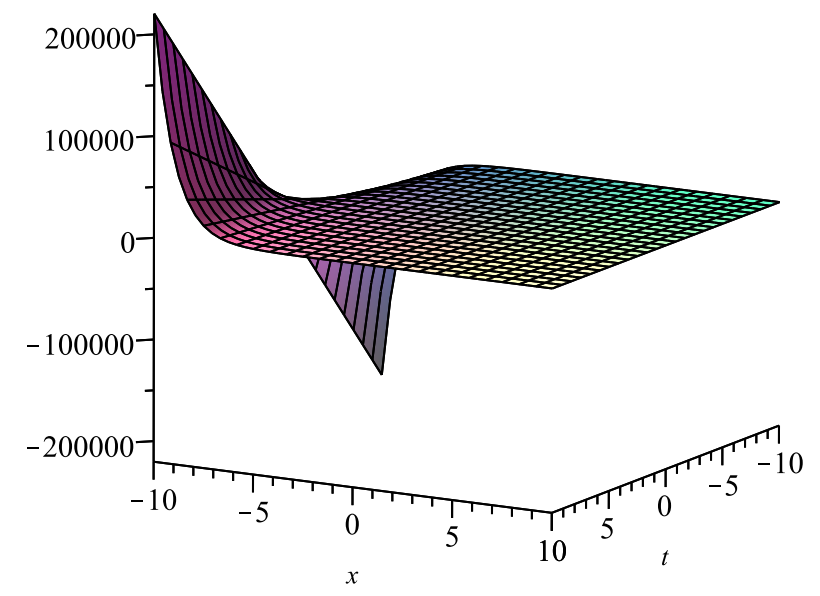

Figure 1: Plot of the approximated solution of the example 4.1.

\section{Evolution Equations}

In this section, we consider three examples of the evolution equations with initial condition by using the mentioned method, Ganji et al [10, and E. Babolian et al [6] have solved these three examples.

Example 5.1. Consider the nonlinear evolution equation [6]

$$
\begin{aligned}
& \phi_{t}-\phi_{x x t}+\left(\frac{\phi^{2}}{2}\right)_{x}=0, \\
& \text { with initial condition } \\
& \phi(x, 0)=x .
\end{aligned}
$$

Taking Laplace transform on both sides of equation (17) and using initial condition (17), it gives

$$
\mathcal{L}[\phi(x, t)]=\frac{1}{s} x+\frac{1}{s} \mathcal{L}\left[\phi_{x x t}-\left(\frac{\phi^{2}}{2}\right)_{x}\right] .
$$

Applying inverse Laplace transform to [18], it gives

$$
\phi(x, t)=x+\mathcal{L}^{-1}\left[\frac{1}{s} \mathcal{L}\left(\phi_{x x t}\right)\right]-\mathcal{L}^{-1}\left[\frac{1}{s} \mathcal{L}\left[\left(\frac{\phi^{2}}{2}\right)_{x}\right]\right] .
$$

We write $\phi(x, t)$ in the form of an infinite series as

$$
\phi(x, t)=\sum_{n=0}^{\infty} \phi_{n}(x, t)
$$

and the nonlinear term $\phi^{2}$ is decomposed by Adomian polynomials as

$$
\phi^{2}=\sum_{n=0}^{\infty} C_{n}(x, t),
$$


where

$$
C_{n}(x, t)=\left.\frac{1}{n !} \frac{d^{n}}{d \lambda^{n}}\left[\sum_{i=0}^{\infty}\left(\lambda^{i} \phi_{i}\right)^{2}\right]\right|_{\lambda=0}
$$

Insert 20 and 21 in 19, we have

$$
\sum_{n=0}^{\infty} \phi_{n}(x, t)=x+\mathcal{L}^{-1}\left[\frac{1}{s} \mathcal{L}\left[\sum_{n=0}^{\infty} \phi_{n, x x t}\right]\right]-\mathcal{L}^{-1}\left[\frac{1}{s} \mathcal{L}\left[\sum_{n=0}^{\infty} C_{n}(x, t)\right]\right] .
$$

Putting values for $n=0,1,2,3, \ldots$, we get

$$
\begin{aligned}
& \phi_{0}(x, t)=x, \\
& \phi_{1}(x, t)=-x t, \\
& \phi_{2}(x, t)=x t^{2}, \\
& \phi_{3}(x, t)=-x t^{3}, \\
& \phi_{4}(x, t)=x t^{4} .
\end{aligned}
$$

Therefore, the solution in the form of series is $\phi(x, t)=x\left[1-t+t^{2}-t^{3}+t^{4}-\ldots\right]$.

Which is the same approximation obtained in [6] by HPM and [10] by VIM.

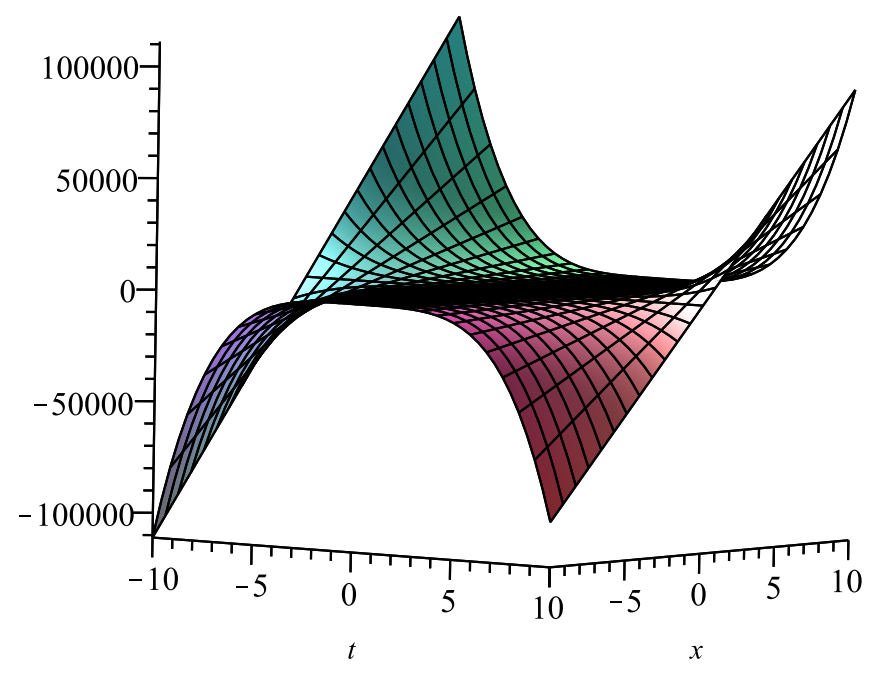

Figure 2: Plot of the approximated solution of the example 5.1.

Example 5.2. Consider the evolution equation [6]

$$
\begin{aligned}
& \phi_{t}+\phi_{x}=2 \phi_{x x t}, \\
& \text { with initial condition } \\
& \phi(x, 0)=\exp (-x) .
\end{aligned}
$$

Taking Laplace transform on both sides of equation (23) and using initial condition (23), it gives

$$
\mathcal{L}[\phi(x, t)]=\frac{1}{s} \exp (-x)-\frac{1}{s} \mathcal{L}\left[\phi_{x}\right]+\frac{2}{s} \mathcal{L}\left[\phi_{x x t}\right]
$$


Applying inverse Laplace transform to 24], it gives

$$
\phi(x, t)=\exp (-x)-\mathcal{L}^{-1}\left[\frac{1}{s} \mathcal{L}\left[\phi_{x}\right]\right]+2 \mathcal{L}^{-1}\left[\frac{1}{s} \mathcal{L}\left[\phi_{x x t}\right]\right] .
$$

We may write series solution $\phi(x, t)$ as

$$
\phi(x, t)=\sum_{n=0}^{\infty} \phi_{n}(x, t)
$$

Putting equation (26) in (25), we have

$$
\sum_{n=0}^{\infty} \phi_{n}(x, t)=\exp (-x)-\mathcal{L}^{-1}\left[\frac{1}{s} \mathcal{L}\left[\sum_{n=0}^{\infty} \phi_{n, x}\right]\right]+2 \mathcal{L}^{-1}\left[\frac{1}{s} \mathcal{L}\left[\sum_{n=0}^{\infty} \phi_{n, x x t}\right]\right] .
$$

Putting values for $n=0,1,2,3, \ldots$, we get

$$
\begin{aligned}
& \phi_{0}(x, t)=\exp (-x) \\
& \phi_{1}(x, t)=\exp (-x) t \\
& \phi_{2}(x, t)=\exp (-x)\left[\frac{t^{2}}{2}+2 t\right] \\
& \phi_{3}(x, t)=\exp (-x)\left[\frac{t^{3}}{3 !}+2 t^{2}+4 t\right] \\
& \phi_{4}(x, t)=\exp (-x)\left[\frac{t^{4}}{4 !}+t^{3}+6 t^{2}+8 t\right] .
\end{aligned}
$$

We obtained the approximated solution as the infinite series of the form

$$
\phi(x, t)=\exp (-x)\left[1+15 t+\frac{17}{2} t^{2}+\frac{7}{6} t^{3}+\frac{1}{24} t^{4}\right]
$$

which is the same approximation obtained in [6] by HPM.

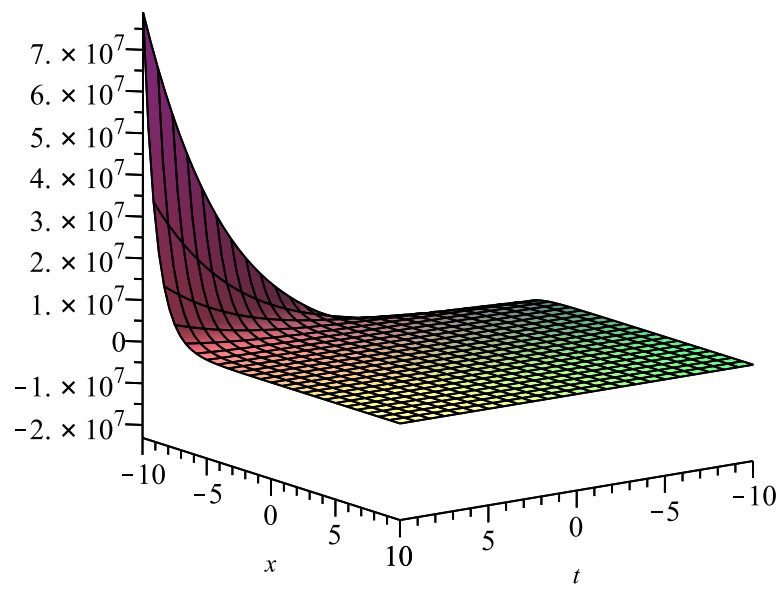

Figure 3: Plot of the approximated solution of the example 5.2 
Example 5.3. Consider the evolution equation [6]

$$
\begin{aligned}
& \phi_{t}-\phi_{x x x x}=0, \\
& \text { with initial condition } \\
& \phi(x, 0)=\sin (x) .
\end{aligned}
$$

Taking Laplace transform on both sides of equation (28) and using initial condition (28), it gives

$$
\mathcal{L}[\phi(x, t)]=\frac{1}{s} \sin (x)+\frac{1}{s} \mathcal{L}\left[\phi_{x x x x}\right]
$$

Applying inverse Laplace transform to (29), we get

$$
\phi(x, t)=\sin (x)+\mathcal{L}^{-1}\left[\frac{1}{s} \mathcal{L}\left[\phi_{x x x x}\right]\right] .
$$

We may write series solution of $\phi(x, t)$ as

$$
\phi(x, t)=\sum_{n=0}^{\infty} \phi_{n}(x, t) .
$$

Putting equation (31) in (30) and inserting values for $n=0,1,2, \ldots$, we obtain

$$
\begin{aligned}
\phi_{0}(x, t) & =\sin (x), \\
\phi_{1}(x, t) & =t \sin (x), \\
\phi_{2}(x, t) & =\frac{t^{2}}{2 !} \sin (x), \\
\phi_{3}(x, t) & =\frac{t^{3}}{3 !} \sin (x), \\
\phi_{4}(x, t) & =\frac{t^{4}}{4 !} \sin (x) .
\end{aligned}
$$

Our approximated solution is

$$
\phi(x, t)=\sin (x)\left[1+t+\frac{t^{2}}{2 !}+\frac{t^{3}}{3 !}+\frac{t^{4}}{4 !}\right] .
$$

Which is the same approximation obtained in [6] by HPM. 


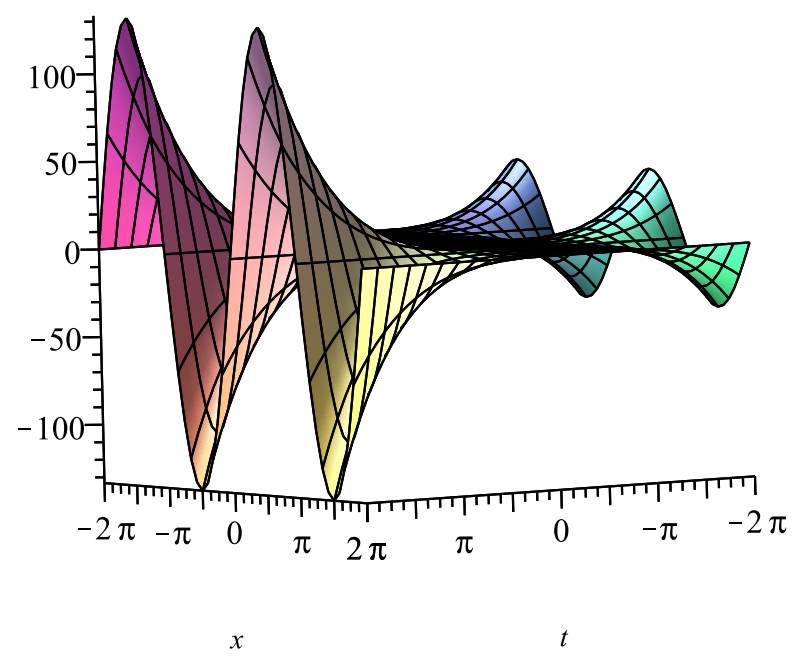

Figure 4: Plot of the approximated solution of the example 5.3 .

\section{Cauchy Reaction-Diffusion Equations}

In this section, we consider four examples of the Cauchy reaction-diffusion equations with initial condition by using the mentioned method, Babolian et.al [6], have solved these three examples by HPM.

Example 6.1. Consider the Cauchy reaction-diffusion equation [6]

$$
\begin{aligned}
& \phi_{t}=\phi_{x x}+2 t \phi, \\
& \text { with initial condition } \\
& \phi(x, 0)=\exp (x) .
\end{aligned}
$$

Applying Laplace transform on left and right sides of equation (32) and using initial condition (32), it gives

$$
\mathcal{L}[\phi(x, t)]=\frac{1}{s} \exp (x)+\frac{1}{s} \mathcal{L}\left[\phi_{x x}+2 t \phi\right] .
$$

Applying inverse Laplace transform to (33), we get

$$
\phi_{n}(x, t)=\exp (x)+\mathcal{L}^{-1}\left[\frac{1}{s} \mathcal{L}\left[\phi_{x x}+2 t \phi\right]\right] .
$$

We may write series solution of $\phi(x, t)$ as

$$
\phi(x, t)=\sum_{n=0}^{\infty} \phi_{n}(x, t) .
$$

Putting equation (34) in (33), and inserting values for $n=0,1,2, \ldots$, we obtain

$$
\begin{aligned}
& \phi_{0}(x, t)=\exp (x), \\
& \phi_{1}(x, t)=\left(t+t^{2}\right) \exp (x), \\
& \phi_{2}(x, t)=\left(\frac{t^{4}}{2}+t^{3}+\frac{t^{2}}{2}\right) \exp (x), \\
& \phi_{3}(x, t)=\left(\frac{t^{5}}{2}+\frac{t^{4}}{2}+\frac{t^{6}}{6}+\frac{t^{3}}{6}\right) \exp (x)
\end{aligned}
$$


and so on. Our approximated solution is

$$
\phi(x, t)=\exp (x)\left[1+t+t^{2}+\frac{t^{4}}{2}+t^{3}+\frac{t^{2}}{2}+\frac{t^{5}}{2}+\frac{t^{4}}{2}+\frac{t^{6}}{6}+\frac{t^{3}}{6}\right] .
$$

We see that the approximated solution obtained up to four terms is close agreement with the exact solution $\phi(x, t)=\exp \left(x+t+t^{2}\right)$.

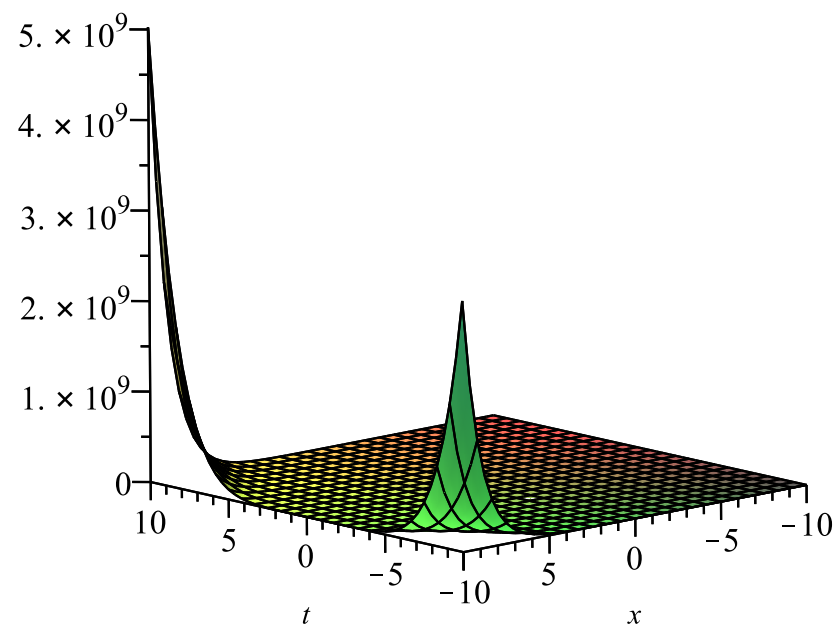

Figure 5: Plot of approximated solution of the example 6.1. 
Example 6.2. Consider the Cauchy reaction-diffusion equation [6]

$$
\begin{aligned}
& \phi_{t}=\phi_{x x}-\left(1+4 x^{2}\right) \phi \\
& \text { corresponding to initial condition } \\
& \phi(x, 0)=\exp \left(x^{2}\right) .
\end{aligned}
$$

Applying Laplace transform on left and right sides of the sides of equation (36) and using initial condition (36), it gives

$$
\mathcal{L}[\phi(x, t)]=\frac{1}{s} \exp \left(x^{2}\right)+\frac{1}{s} \mathcal{L}\left[\phi_{x x}-\left(1+4 x^{2}\right) \phi\right]
$$

Applying inverse Laplace transform to (37), we get

$$
\phi_{n}(x, t)=\exp \left(x^{2}\right)+\mathcal{L}^{-1}\left[\frac{1}{s} \mathcal{L}\left[\phi_{x x}-\left(1+4 x^{2}\right) \phi\right]\right]
$$

We may write series solution of $\phi(x, t)$ as

$$
\phi(x, t)=\sum_{n=0}^{\infty} \phi_{n}(x, t) .
$$

Putting equation (39) in (38) and inserting values for $n=0,1,2, \ldots$, we obtain

$$
\begin{aligned}
\phi_{0}(x, t) & =\exp \left(x^{2}\right) \\
\phi_{1}(x, t) & =-t \exp \left(x^{2}\right), \\
\phi_{2}(x, t) & =\frac{1}{2 !} t^{2} \exp \left(x^{2}\right), \\
\phi_{3}(x, t) & =-\frac{1}{3 !} t^{3} \exp \left(x^{2}\right), \\
\phi_{4}(x, t) & =\frac{1}{4 !} t^{4} \exp \left(x^{2}\right), \\
\phi_{5}(x, t) & =-\frac{1}{5 !} t^{5} \exp \left(x^{2}\right)
\end{aligned}
$$

and so on. Our approximated solution is

$$
\phi(x, t)=\exp \left(x^{2}\right)\left[1-t+\frac{1}{2 !} t^{2}-\frac{1}{3 !} t^{3}+\frac{1}{4 !} t^{4}-\frac{1}{5 !} t^{5}\right]
$$

We see that approximated terms are close to the exact solution

$$
\phi(x, t)=\exp \left(x^{2}+t\right)
$$




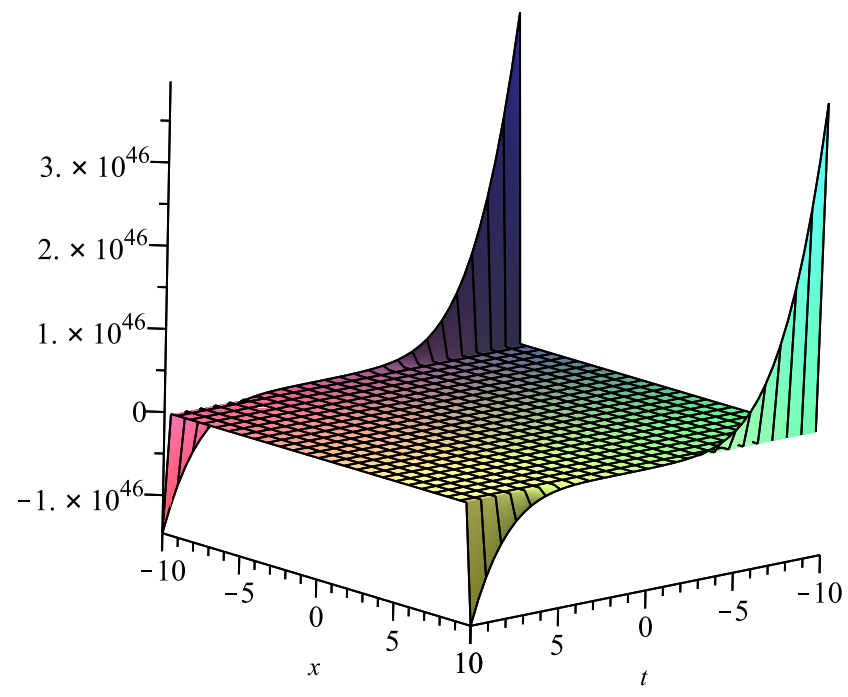

Figure 6: Plot of the approximated solution of the example 6.2.

Example 6.3. Consider the Cauchy reaction-diffusion equation [6]

$$
\begin{aligned}
& \phi_{t}=\phi_{x x}-\left(4 x^{2}-2 t+2\right) \phi, \\
& \text { corresponding to initial condition } \\
& \phi(x, 0)=\exp \left(x^{2}\right) .
\end{aligned}
$$

Applying Laplace transform on left and right sides of equation (40) and using initial condition 40], it gives

$$
\mathcal{L}[\phi(x, t)]=\frac{1}{s} \exp \left(x^{2}\right)+\frac{1}{s} \mathcal{L}\left[\phi_{x x}-\left(4 x^{2}-2 t+2\right) \phi\right] .
$$

Applying inverse Laplace transform to [40, we get

$$
\phi_{n}(x, t)=\exp \left(x^{2}\right)+\mathcal{L}^{-1}\left[\frac{1}{s} \mathcal{L}\left[\phi_{x x}-\left(4 x^{2}-2 t+2\right)\right] \phi\right] .
$$

We can write solution $\phi(x, t)$ in an infinite series as

$$
\phi(x, t)=\sum_{n=0}^{\infty} \phi_{n}(x, t)
$$

Putting equation 43] in 42, and inserting values for $n=0,1,2, \ldots$, we obtain

$$
\begin{aligned}
& \phi_{0}(x, t)=\exp \left(x^{2}\right), \\
& \phi_{1}(x, t)=t^{2} \exp \left(x^{2}\right), \\
& \phi_{2}(x, t)=\frac{1}{2 !} t^{4} \exp \left(x^{2}\right), \\
& \phi_{3}(x, t)=\frac{1}{3 !} t^{6} \exp \left(x^{2}\right), \\
& \phi_{4}(x, t)=\frac{1}{4 !} t^{8} \exp \left(x^{2}\right)
\end{aligned}
$$


and so on. Our approximated solution is

$$
\phi(x, t)=\exp \left(x^{2}\right)\left[1+t^{2}+\frac{1}{2 !} t^{4}+\frac{1}{3 !} t^{6}+\frac{1}{4 !} t^{8}\right]
$$

We see that the approximated solution obtained using LADM up to first five terms is close agreement with the exact solution

$$
\phi(x, t)=\exp \left(x^{2}+t^{2}\right)
$$

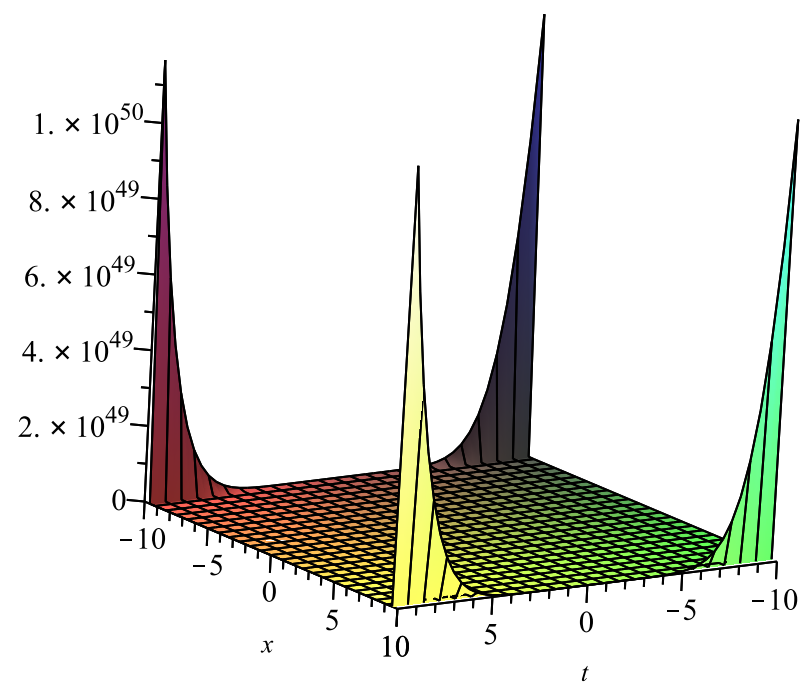

Figure 7: Plot of the approximated solution of the example 6.3 .

Example 6.4. Consider the Cauchy reaction-diffusion equation [6]

$$
\begin{aligned}
& \phi_{t}=\phi_{x x}-\phi, \\
& \text { with initial condition } \\
& \phi(x, 0)=\exp (-x)+x .
\end{aligned}
$$

Applying Laplace transform on left and right sides of the sides of equation (44) and using initial condition, it gives

$$
\mathcal{L}[\phi(x, t)]=\frac{1}{s}[\exp (-x)+x]+\frac{1}{s} \mathcal{L}\left[\phi_{x x}-\phi\right] .
$$

Applying inverse Laplace transform to 45, we get

$$
\phi_{n}(x, t)=\exp (-x)+x+\mathcal{L}^{-1}\left[\frac{1}{s} \mathcal{L}\left[\phi_{x x}-\phi\right]\right] .
$$

We write $\phi(x, t)$ in the form of an infinite series as

$$
\phi(x, t)=\sum_{n=0}^{\infty} \phi_{n}(x, t) .
$$


Putting equation (47) in (46) and inserting values for $n=0,1,2, \ldots$, we obtain

$$
\begin{aligned}
\phi_{0}(x, t) & =\exp (-x)+x, \\
\phi_{1}(x, t) & =-x t, \\
\phi_{2}(x, t) & =\frac{x t^{2}}{2 !}, \\
\phi_{3}(x, t) & =\frac{-x t^{3}}{3 !}, \\
\phi_{4}(x, t) & =\frac{x t^{4}}{4 !}
\end{aligned}
$$

and so on. Our approximated solution is

$$
\phi(x, t)=\exp (-x)+x-x t+\frac{x t^{2}}{2 !}-\frac{x t^{3}}{3 !}+\frac{x t^{4}}{4 !} .
$$

We see that the approximated solution obtained up to five terms is close to the exact solution

$$
\phi(x, t)=\exp (-x)+x \exp (-t) .
$$

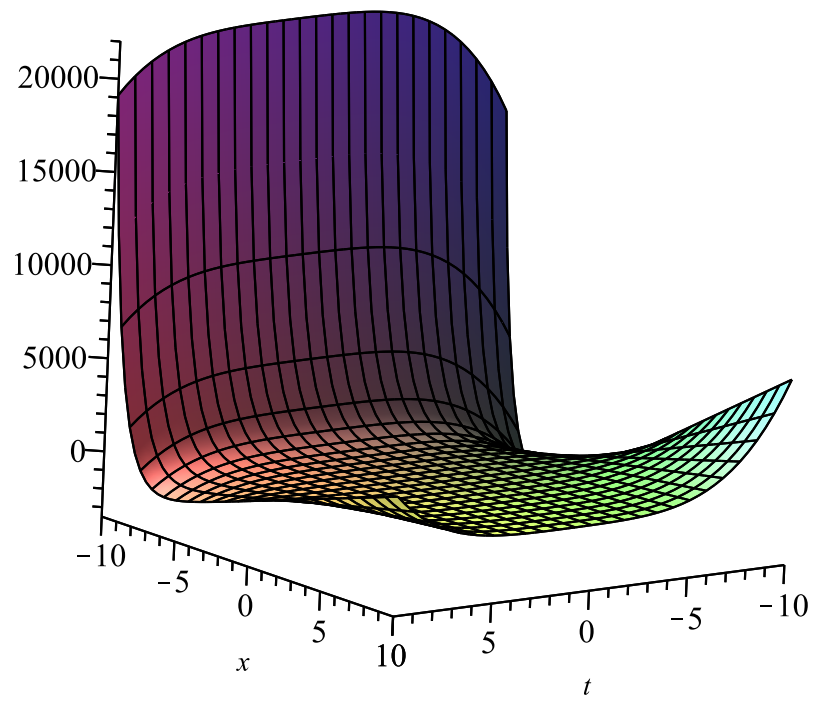

Figure 8: Plot of the approximated solution of the example 6.4.

\section{$7 \quad$ Klien-Gordon Equations}

In this section, we consider five examples of the Klien-Gordon equations with initial condition by using the mentioned method, Babolian et.al [6], have solved these three examples by HPM.

Example 7.1. Consider the linear Klien-Gordon equation [6]

$$
\begin{aligned}
& \phi_{t t}-\phi_{x x}=\phi \\
& \text { with initial conditions } \\
& \phi(x, 0)=1+\sin (x), \phi_{t}(x, 0)=0 .
\end{aligned}
$$


Applying Laplace transform to left and right sides of the equation 48) and using initial condition (48), it gives

$$
\mathcal{L}[\phi(x, t)]=\frac{1}{s}[1+\sin (x)]+\frac{1}{s^{2}} \mathcal{L}\left[\phi_{x x}+\phi\right]
$$

Upon using inverse Laplace transform to 49, we get

$$
\phi_{n}(x, t)=1+\sin (x)+\mathcal{L}^{-1}\left[\frac{1}{s^{2}} \mathcal{L}\left[\phi_{x x}+\phi\right]\right] .
$$

We write $\phi(x, t)$ in the form of an infinite series as

$$
\phi(x, t)=\sum_{n=0}^{\infty} \phi_{n}(x, t) .
$$

Putting equation (51) in 50 and inserting values for $n=0,1,2, \ldots$, we obtain

$$
\begin{aligned}
\phi_{0}(x, t) & =1+\sin (x), \\
\phi_{1}(x, t) & =\frac{t^{2}}{2 !}, \\
\phi_{2}(x, t) & =\frac{t^{4}}{4 !}, \\
\phi_{3}(x, t) & =\frac{t^{6}}{6 !}, \\
\phi_{4}(x, t) & =\frac{t^{8}}{8 !}
\end{aligned}
$$

and so on. Our approximated solution is

$$
\phi(x, t)=\sin (x)+1+\frac{t^{2}}{2 !}+\frac{t^{4}}{4 !}+\frac{t^{6}}{6 !}+\frac{t^{8}}{8 !} .
$$

We see that the approximated solution received by LADM has close agreement to the exact solution

$$
\phi(x, t)=\sin (x)+\cosh (t) .
$$




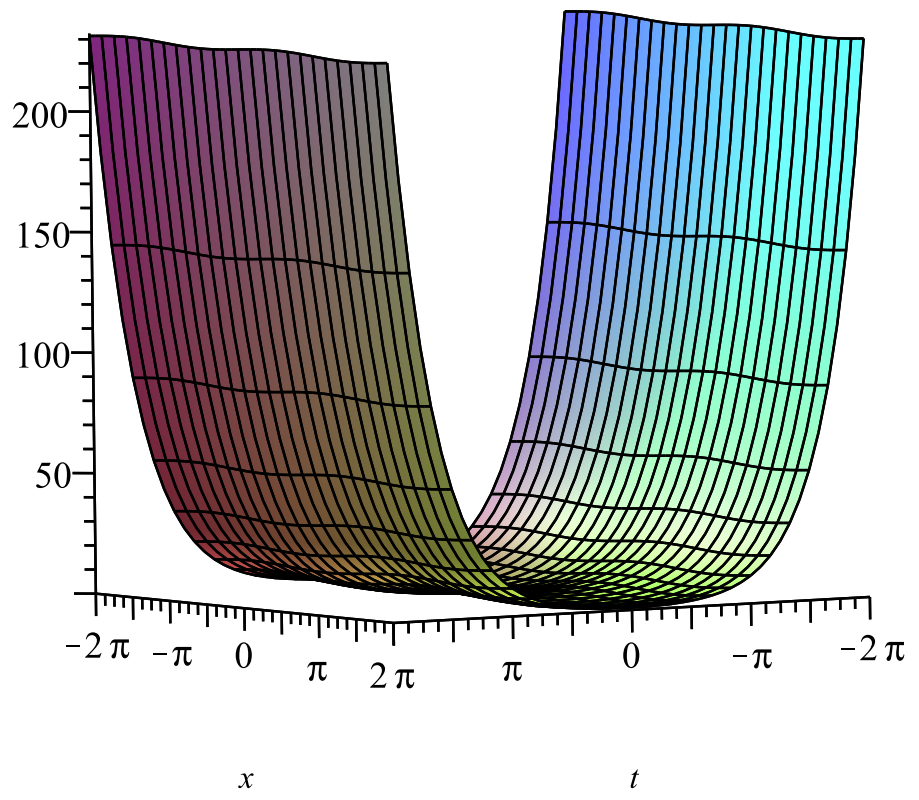

Figure 9: Plot of the approximated solution of the example 7.1.

Example 7.2. Consider the nonlinear Klien-Gordon equation [8]

$$
\begin{aligned}
& \phi_{t t}-\phi_{x x}-\phi^{2}=2 x^{2}-2 t^{2}+x^{4} t^{4}, \\
& \text { with initial conditions } \\
& \phi(x, 0)=0, \phi_{t}(x, 0)=0 .
\end{aligned}
$$

Taking Laplace transform on both sides of equation (52) and using initial condition (52), it gives

$$
\mathcal{L}[\phi(x, t)]=\frac{1}{s^{2}} \mathcal{L}\left[2 x^{2}-2 t^{2}+x^{4} t^{4}\right]+\frac{1}{s^{2}} \mathcal{L}\left[\phi_{x x}\right]+\frac{1}{s^{2}} \mathcal{L}\left[\phi^{2}\right] .
$$

Applying inverse Laplace transform to 53 , we get

$$
\phi(x, t)=x^{2} t^{2}-2 \mathcal{L}^{-1}\left[\frac{1}{s^{2}} \mathcal{L}\left[t^{2}\right]\right]+x^{4} \mathcal{L}^{-1}\left[\frac{1}{s^{2}} \mathcal{L}\left[t^{4}\right]\right]+\mathcal{L}^{-1}\left[\frac{1}{s^{2}} \mathcal{L}\left[\phi_{x x}\right]\right]+\mathcal{L}^{-1}\left[\frac{1}{s^{2}} \mathcal{L}\left[\phi^{2}\right]\right] .
$$

We write $\phi(x, t)$ in the form of an infinite series as

$$
\phi(x, t)=\sum_{n=0}^{\infty} \phi_{n}(x, t)
$$

and the nonlinear term $\phi^{2}$ is defined by Adomian polynomials as

$$
\phi^{2}=\sum_{n=0}^{\infty} D_{n}(x, t)
$$

where

$$
D_{n}(x, t)=\left.\frac{1}{n !} \frac{d^{n}}{d \lambda^{n}}\left[\sum_{i=0}^{\infty}\left(\lambda^{i} \phi_{i}\right)^{2}\right]\right|_{\lambda=0}
$$


Insert (55) and (56) in (54), we have

$$
\sum_{n=0}^{\infty} \phi_{n}(x, t)=x^{2} t^{2}-\mathcal{L}^{-1}\left[\frac{1}{s^{2}} \mathcal{L}\left[2 t^{2}+x^{4} t^{4}\right]\right]+\mathcal{L}^{-1}\left[\frac{1}{s^{2}} \mathcal{L}\left[\sum_{n=0}^{\infty}\left[\phi_{n, x x}(x, t)+D_{n}(x, t)\right]\right]\right.
$$

Putting values for $n=0,1,2, \ldots$, we obtain

$$
\begin{aligned}
& \phi_{0}(x, t)=x^{2} t^{2}, \\
& \phi_{1}(x, t)=0, \\
& \phi_{2}(x, t)=0 .
\end{aligned}
$$

All the next terms are vanish for $n \geq 3$, therefore the approximated solution is close to the exact solution [8], given as below

$$
\phi(x, t)=x^{2} t^{2}
$$

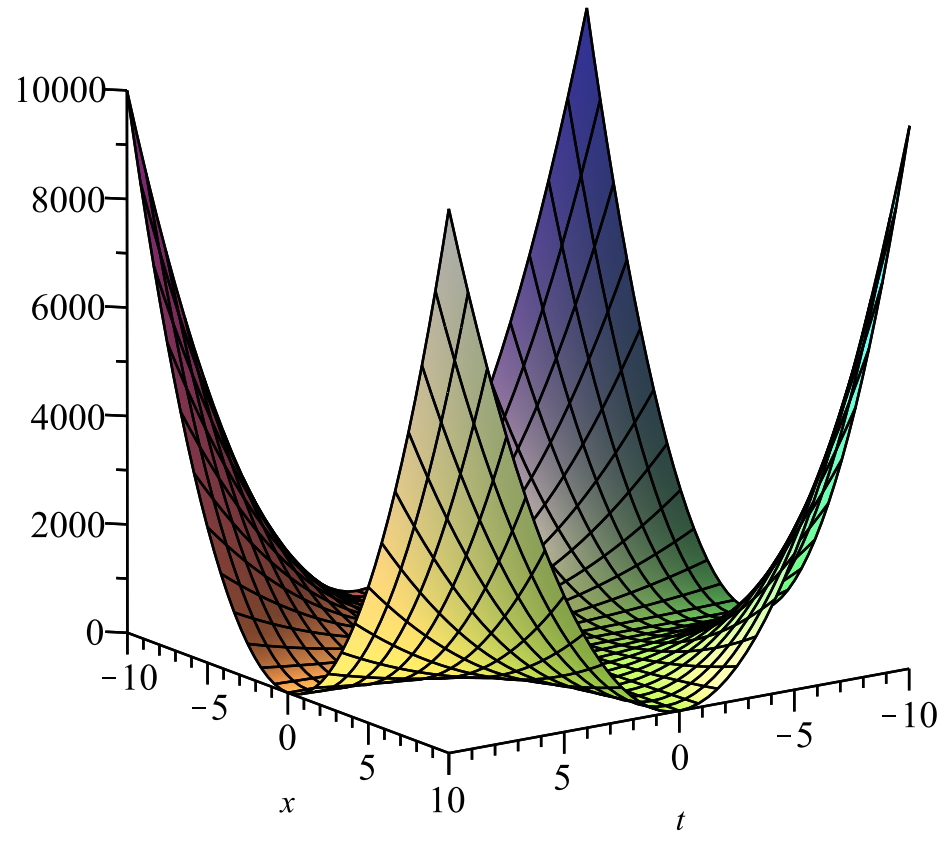

Figure 10: Plot of the approximated solution of the example 7.2 . 
Example 7.3. Consider the linear Klien-Gordon equation [6]

$$
\begin{aligned}
& \phi_{t t}-\phi_{x x}-2 \phi=2 \sin (x) \sin (t), \\
& \text { with initial conditions } \\
& \phi(x, 0)=0, \phi_{t}(x, 0)=\sin (x) .
\end{aligned}
$$

Taking Laplace transform on both sides of equation (58) and using initial condition (58), it gives

$$
\mathcal{L}[\phi(x, t)]=\frac{1}{s^{2}} \sin (x)+\frac{1}{s^{2}} \mathcal{L}\left[\phi_{x x}+2 \phi+2 \sin (x) \sin (t)\right]
$$

Using inverse Laplace transform to 59 , we get

$$
\phi_{n}(x, t)=t \sin (x)+\mathcal{L}^{-1}\left[\frac{1}{s^{2}} \mathcal{L}\left[\phi_{x x}\right]\right]+\mathcal{L}^{-1}\left[\frac{1}{s^{2}} \mathcal{L}[2 \phi]\right]+2 \sin (x) \mathcal{L}^{-1}\left[\frac{1}{s^{2}} \mathcal{L}[\sin (t)]\right]
$$

We write $\phi(x, t)$ in the form of an infinite series as

$$
\phi(x, t)=\sum_{n=0}^{\infty} \phi_{n}(x, t)
$$

Putting equation 61 in 60 and inserting values for $n=0,1,2, \ldots$, we obtain

$$
\begin{aligned}
& \phi_{0}(x, t)=t \sin (x) \\
& \phi_{1}(x, t)=\left[\frac{t^{3}}{3 !}-2 t+2 \sin (t)\right] \sin (x) \\
& \phi_{2}(x, t)=\left[\frac{t^{5}}{5 !}-\frac{2 t^{3}}{3 !}+2 t-2 \sin (t)\right] \sin (x), \\
& \phi_{3}(x, t)=\left[\frac{t^{7}}{7 !}-\frac{2 t^{5}}{5 !}+\frac{2 t^{3}}{3 !}-2 t+2 \sin (t)\right] \sin (x), \\
& \phi_{4}(x, t)=\left[\frac{t^{9}}{9 !}+\frac{2 t^{7}}{7 !}+\frac{2 t^{5}}{5 !}-\frac{2 t^{3}}{3 !}+2 t-2 \sin (t)\right] \sin (x)
\end{aligned}
$$

and so on. We see that the approximated solution obtained by LADM up to five terms is close to the exact solution given by

$$
\phi(x, t)=\sin (x) \sin (t)
$$




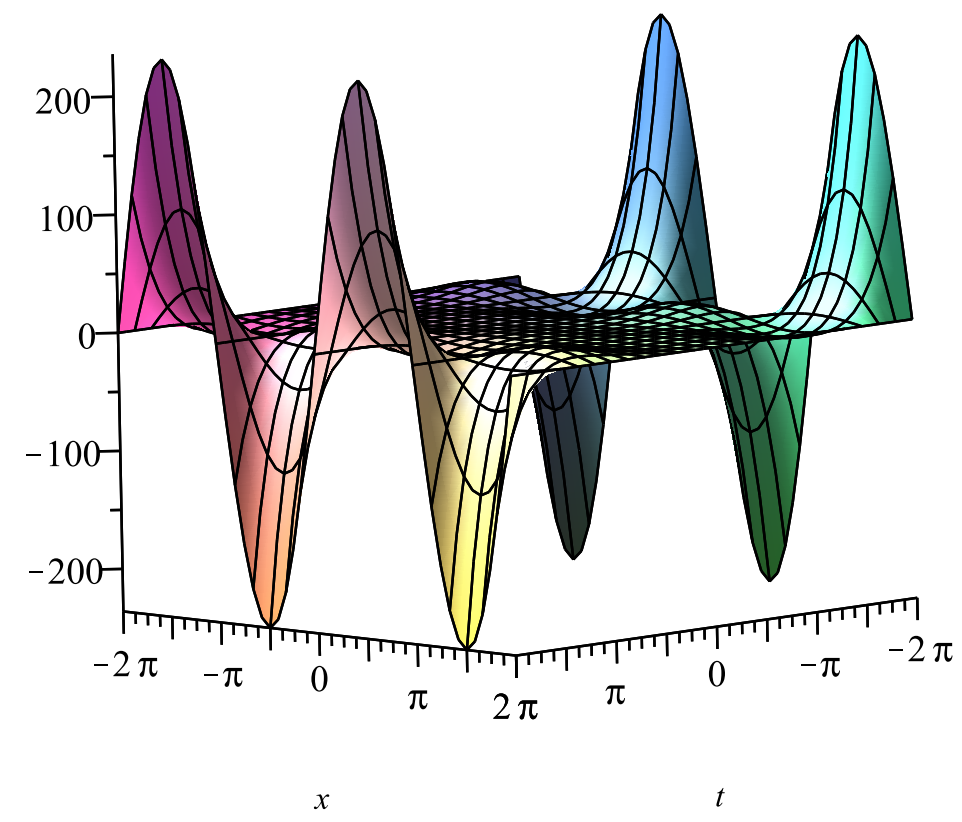

Figure 11: Plot of the approximated solution of the example 7.3 .

Example 7.4. Consider the nonlinear Klien-Gorden equation [16]

$$
\begin{aligned}
& \phi_{t t}-\phi_{x x}+\phi^{2}=0, \\
& \text { corresponding to the initial conditions } \\
& \phi(x, 0)=1+\sin (x), \phi_{t}(x, 0)=0 .
\end{aligned}
$$

Taking Laplace transform on both sides of equation (62) and using initial condition (62), it gives

$$
\mathcal{L}[\phi(x, t)]=\frac{1}{s}[1+\sin (x)]+\frac{1}{s^{2}}\left[\mathcal{L}\left[\phi_{x x}-\phi^{2}\right]\right] .
$$

Applying inverse Laplace transform to 63), we get

$$
\phi(x, t)=1+\sin (x)+\mathcal{L}^{-1}\left[\frac{1}{s^{2}} \mathcal{L}\left[\phi_{x x}\right]\right]-\mathcal{L}^{-1}\left[\frac{1}{s^{2}} \mathcal{L}\left[\phi^{2}\right]\right] .
$$

We write $\phi(x, t)$ in the form of an infinite series as

$$
\phi(x, t)=\sum_{n=0}^{\infty} \phi_{n}(x, t)
$$

and the nonlinear term $\phi^{2}$ be defined by Adomian polynomials as

$$
\phi^{2}=\sum_{n=0}^{\infty} P_{n}(x, t)
$$

where

$$
P_{n}(x, t)=\left.\frac{1}{n !}\left[\frac{d^{n}}{d \lambda^{n}}\left(\sum_{i=0}^{\infty}\left(\lambda^{i} \phi_{i}\right)\right)\left(\sum_{i=0}^{\infty}\left(\lambda^{i} \phi_{i, x}\right)\right)\right]\right|_{\lambda=0} .
$$


Insert 65 and 66 in 64, we have

$$
\sum_{n=0}^{\infty} \phi_{n}(x, t)=1+\sin (x)+\mathcal{L}^{-1}\left[\frac{1}{s^{2}} \mathcal{L}\left[\sum_{n=0}^{\infty} \phi_{n, x x}(x, t)\right]\right]-\mathcal{L}^{-1}\left[\frac{1}{s^{2}} \mathcal{L}\left[\sum_{n=0}^{\infty} P_{n}(x, t)\right]\right]
$$

Putting values for $n=0,1,2, \ldots$, we obtain

$$
\begin{aligned}
& \phi_{0}(x, t)=1+\sin (x) \\
& \phi_{1}(x, t)=\frac{t^{2}}{2 !}\left[1-3 \sin (x)-\sin ^{2}(x)\right] \\
& \phi_{2}(x, t)=\frac{t^{4}}{4 !}\left[-2-5 \sin (x)-8 \sin ^{2}(x)-2 \sin ^{3}(x)+2 \cos (2 x)\right]
\end{aligned}
$$

and so on. Thus, the approximated solution is expressed as

$$
\begin{aligned}
& \phi(x, t)=1+\sin (x)+\frac{t^{2}}{2 !}\left(1-3 \sin (x)-\sin ^{2}(x)\right) \\
& +\frac{t^{4}}{4 !}\left(-2-5 \sin (x)-8 \sin ^{2}(x)-2 \sin ^{3}(x)+2 \cos (2 x)\right)+\ldots
\end{aligned}
$$

Which is the exactly same approximated solution obtained in [16] by DTM.

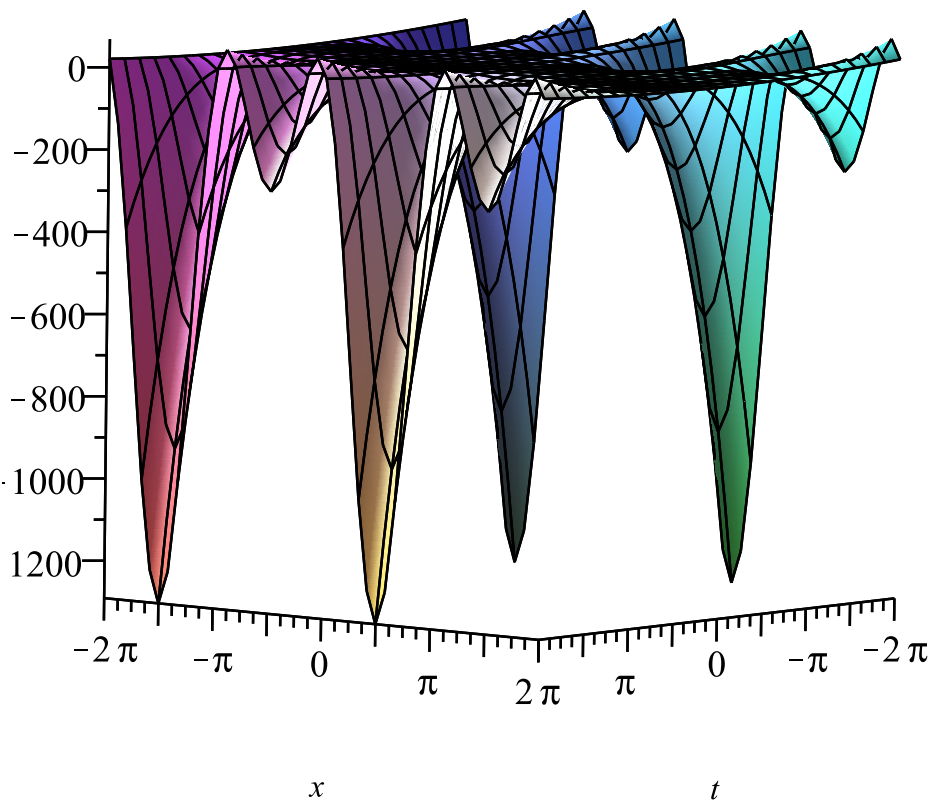

Figure 12: Plot of the approximated solution of the example 7.4 
Example 7.5. Consider the nonlinear Klien-Gorden equation [16]

$$
\begin{aligned}
& \phi_{t t}-\phi_{x x}+\phi^{2}=6 x^{3} t-6 x t^{3}+x^{6} t^{6}, \\
& \text { with the given conditions } \\
& \phi(x, 0)=0, \phi_{t}(x, 0)=0 .
\end{aligned}
$$

Using Laplace transform to 69 and using initial condition 69, it gives

$$
\mathcal{L}[\phi(x, t)]=\frac{1}{s^{2}} \mathcal{L}\left[6 x^{3} t\right]-\frac{1}{s^{2}} \mathcal{L}\left[6 x t^{3}\right]+\frac{1}{s^{2}} \mathcal{L}\left[x^{6} t^{6}\right]+\frac{1}{s^{2}} \mathcal{L}\left[\phi_{x x}\right]-\frac{1}{s^{2}} \mathcal{L}\left[\phi^{2}\right]
$$

Applying inverse Laplace transform to 70 , we get

$$
\phi(x, t)=x^{3} t^{3}-6 x \mathcal{L}^{-1}\left[\frac{1}{s^{2}} \mathcal{L}\left[t^{3}\right]\right]+x^{6} \mathcal{L}^{-1}\left[\frac{1}{s^{2}} \mathcal{L}\left[t^{6}\right]\right]+\mathcal{L}^{-1}\left[\frac{1}{s^{2}} \mathcal{L}\left[\phi_{x x}\right]\right]-\mathcal{L}^{-1}\left[\frac{1}{s^{2}} \mathcal{L}\left[\phi^{2}\right]\right]
$$

We write $\phi(x, t)$ in the form of an infinite series as

$$
\phi(x, t)=\sum_{n=0}^{\infty} \phi_{n}(x, t)
$$

and the nonlinear term $\phi^{2}$ be defined by Adomian polynomials as

$$
\phi^{2}=\sum_{n=0}^{\infty} P_{n}(x, t)
$$

where

$$
P_{n}(x, t)=\left.\frac{1}{n !}\left[\frac{d^{n}}{d \lambda^{n}}\left(\sum_{i=0}^{\infty}\left(\lambda^{i} \phi_{i}\right)\right)\left(\sum_{i=0}^{\infty}\left(\lambda^{i} \phi_{i, x}\right)\right)\right]\right|_{\lambda=0} .
$$

Putting equations 72 and 73 in 71 .

$$
\begin{aligned}
& \phi_{n}(x, t)=x^{3} t^{3}-6 x \mathcal{L}^{-1}\left[\frac{1}{s^{2}} \mathcal{L}\left[t^{3}\right]\right]+x^{6} \mathcal{L}^{-1}\left[\frac{1}{s^{2}} \mathcal{L}\left[t^{6}\right]\right]+\mathcal{L}^{-1}\left[\frac{1}{s^{2}} \mathcal{L}\left[\sum_{n=0}^{\infty} \phi_{n, x x}\right]\right] \\
& -\mathcal{L}^{-1}\left[\frac{1}{s^{2}} \mathcal{L}\left[\sum_{n=0}^{\infty} P_{n}(x, t)\right]\right] .
\end{aligned}
$$

Putting values for $n=0,1,2, \ldots$, it gives

$$
\begin{aligned}
& \phi_{0}(x, t)=x^{3} t^{3}, \\
& \phi_{1}(x, t)=0, \\
& \phi_{2}(x, t)=0 .
\end{aligned}
$$

All the next terms are vanishing for $n \geq 3$, therefore the approximated solution is close to the exact the solution [16] given by

$$
\phi(x, t)=x^{3} t^{3}
$$

We see that the approximated solution obtained up to five terms has close agreement with the exact solution

$$
\phi(x, t)=x^{3} t^{3}
$$

Which is the same solution obtained in [16] by HPM. 


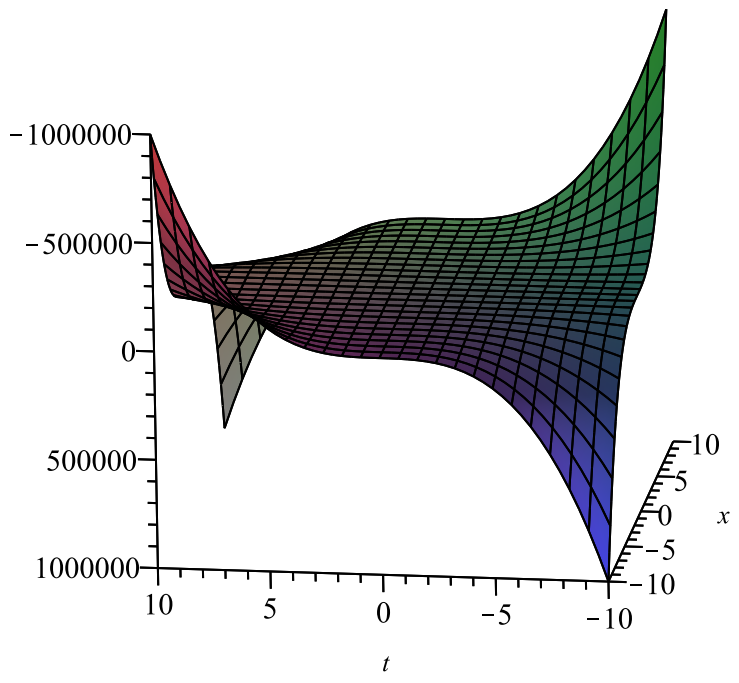

Figure 13: Plot of the approximated solution of the example 7.5

Example 7.6. Consider the nonlinear Fisher's equation [15]

$$
\begin{aligned}
& \phi_{t}-\phi_{x x}-\phi+\phi^{2}=0, \\
& \text { with initial condition } \\
& \phi(x, 0)=\beta .
\end{aligned}
$$

Taking Laplace transform on both sides of equation 75 and using initial condition (75), it gives

$$
\mathcal{L}[\phi(x, t)]=\frac{1}{s} \beta+\frac{1}{s} \mathcal{L}\left[\phi_{x x}\right]+\frac{1}{s} \mathcal{L}[\phi]-\frac{1}{s} \mathcal{L}\left[\phi^{2}\right] .
$$

Applying inverse Laplace transform to $(76)$, we get

$$
\phi(x, t)=\beta+\mathcal{L}^{-1}\left[\frac{1}{s} \mathcal{L}\left[\phi_{x x}\right]\right]+\mathcal{L}^{-1}\left[\frac{1}{s} \mathcal{L}[\phi]\right]-\mathcal{L}^{-1}\left[\frac{1}{s} \mathcal{L}\left[\phi^{2}\right]\right] .
$$

We write $\phi(x, t)$ in the form of an infinite series as

$$
\phi(x, t)=\sum_{n=0}^{\infty} \phi_{n}(x, t)
$$

and the nonlinear term $\phi^{2}$ be defined by Adomian polynomials as

$$
\phi^{2}=\sum_{n=0}^{\infty} H_{n}(x, t)
$$

where

$$
H_{n}(x, t)=\frac{1}{n !}\left[\frac{d^{n}}{d \lambda^{n}}\left(\sum_{i=0}^{\infty}\left(\lambda^{i} \phi_{i}\right)\right)\left(\sum_{i=0}^{\infty}\left(\lambda^{i} \phi_{i, x}\right)\right)\right]_{\lambda=0} .
$$

Putting equations $(78)$ and 790 in (77), it gives

$$
\sum_{i=0}^{\infty} \phi_{n}(x, t)=\beta+\mathcal{L}^{-1}\left[\frac{1}{s} \mathcal{L}\left[\sum_{i=0}^{\infty} \phi_{n, x x}\right]\right]+\mathcal{L}^{-1}\left[\frac{1}{s} \mathcal{L}\left[\sum_{i=0}^{\infty} \phi_{n}\right]\right]-\mathcal{L}^{-1}\left[\frac{1}{s} \mathcal{L}\left[\sum_{i=0}^{\infty} H_{n}(x, t)\right]\right] .
$$


Inserting the values for $n=0,1,2, \ldots$, we obtain

$$
\begin{aligned}
& \phi_{0}(x, t)=\beta \\
& \phi_{1}(x, t)=\left(\beta-\beta^{2}\right) t \\
& \phi_{2}(x, t)=\left(\beta-3 \beta^{2}+2 \beta^{3}\right) \frac{t^{2}}{2 !}, \\
& \phi_{3}(x, t)=\left(\beta-7 \beta^{2}+12 \beta^{3}-6 \beta^{4}\right) \frac{t^{3}}{3 !}, \\
& \phi_{4}(x, t)=\left(\beta-25 \beta^{2}+114 \beta^{3}-23 \beta^{4}-84 \beta^{5}\right) \frac{t^{4}}{4 !}
\end{aligned}
$$

and so on. We see that the approximated solutions obtained up to five terms has close agreement with the exact solution given by [15]

$$
\phi(x, t)=\frac{\beta \exp (t)}{1-\beta+\beta \exp (t)} .
$$

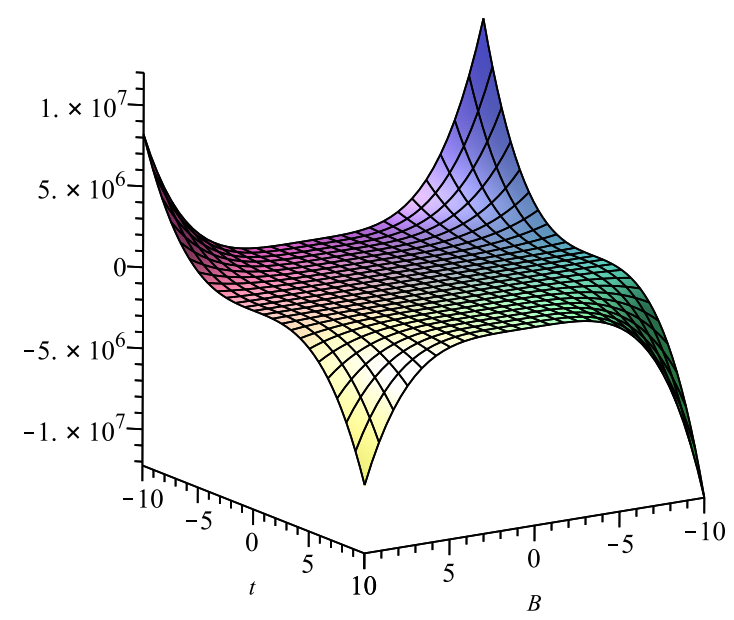

Figure 14: Plot of the approximated solution of the example 7.6 .

\section{Conclusion}

In this study, we have successfully applied the Laplace Adomian decomposition method (LADM) to linear and nonlinear PDEs with initial conditions to find the approximated solutions. As a conclusion, we see that the approximate solutions obtained by adopted method LADM has close agreement to the exact solutions. Maple 2016 has been used for graphical visualization in this paper.

Acknowledgments: I am really thankful to the reviewers for their constructive comments which improved this paper very well.

\section{References}

[1] Adomian G (1995) Delayed nonlinear dynamical systems. Mathematical and Computer Modelling, 22(3): $77-79$. 
[2] Adomian G (1995) Random Volterra integral equations. Mathematical and Computer Modelling, 22(8): $101-102$

[3] Adomian G (1995) Solving the mathematical models of neurosciences and medicine. Mathematics and Computers in Simulation, 40: 107-114.

[4] Adomian G (1995) The Nikolaevskiy model for nonlinear seismic waves. Mathematical and Computer Modelling, 22(3): 81-82.

[5] Andrianov I, Olevskii IV, Tokarzewski S (1998) A modified Adomian's decomposition method. Appl. Math. Mech, 62(2): 309-314.

[6] Babolian E, Azizi A, Saeidian J (2009) Some notes on using the homotopy perturbation method for solving time-dependent differential equations. Mathematical and Computer Modeling, 50: 213-224.

[7] Dehghan M, Manafian J, Saadatmandi A (2010) Solving nonlinear fractional partial differential equations using the homotopy analysis method. Num. Meth. Partial Diff. Equns, 26(2): 448-479.

[8] Dhunde RR, Waghmare GL (2016) Analytical Solution of the Nonlinear Klein-Gordon Equation using Double Laplace Transform and Iterative Method. American J. Comput. Appl. Math, 6(6): 195-201.

[9] Elgazery NS (2008) Numerical solution for the Falkner-Skan equation. Chaos Solitons and Fractals, 35(4): $738-746$.

[10] Ganji DD, Tari H, Joobari MB (2007) Variational iteration method and homotopy perturbation method for nonlinear evolution equations.

[11] Haq F, Shah K, Rahman G, Li Y, Shahzad M (2018) Computational Analysis of Complex Population Dynamical Model with Arbitrary Order. Complexity, 2018: 8 pages.

[12] Hu Y, Luo Y, Lu Z (2008) Analytical solution of the linear fractional differential equation by Adomian decomposition method. J.Comput.Appl.Math, 215: 220-229.

[13] Hussain M, Khan M (2010) Modified Laplace Decomposition Method. Applied Mathematical Sciences, 36: $1769-1783$.

[14] Khalil H, Khan RA (2014) A new method based on Legendre polynomials for solutions of the fractional twodimensional heat conduction equation. Comput.Math. Appl, 67: 1938-1953.

[15] Mohyud-Din ST, Noor MA (2009) The HPM for Solving Partial Differential Equations. Applied Mathematical Sciences, 64: 157-170.

[16] Ravi Kanth ASV, Aruna K (2009) Differential transform method for solving the linear and nonlinear Klien-Gordon equation. Computer Physics Communications, 180: 708-711.

[17] Shah K, Bushnaq S (2017) Numerical treatment of fractional endemic disease model via Laplace Adomian decomposition method. Journal of Science and Arts, 2(39): 257-268.

[18] Shah K, Khalil H, Khan RA (2016) Analytical Solutions of Fractional Order Diffusion Equations by Natural Transform Method. Iran Journal of Science and Technology (Trans Sci:A), 2016: 14 pages. Int. J. Comput. Math. Appl, 54: 1018-1027.

[19] Tatari M, Dehghan M (2005) Numerical solution of Laplace equation in a disk using the Adomian decomposition method. Phys. Scr, 72(5): 345-348.

[20] Wazwaz A (2006) The modified decomposition method for analytic treatment of differential equations. Applied Mathematics and Computation, 173: 165-176.

[21] Yang AM, Zhang YZ, Long Y (2013) Te Yang-Fourier transforms to heat-conduction in a semi-infnite fractal bar. Termal Science, 17(3): 707-713. 